\title{
Update on the clinical utility of fenofibrate in mixed dyslipidemias: mechanisms of action and rational prescribing
}

\author{
Michel Farnier \\ Point Médical, Dijon, France
}

Correspondence: Michel Farnier Point Médical, Rond Point de la Nation, 21000 Dijon, France

Tel +333807038 I3

$\mathrm{Fax}+33380703894$

Email michelfarnier@nerim.net

\begin{abstract}
Mixed dyslipidemia is a common lipid disorder characterized by the presence of an atherogenic lipoprotein phenotype due to abnormalities in various atherogenic and antiatherogenic lipoproteins. Despite the link between the decrease of LDL-cholesterol by statin treatment and the prevention of cardiovascular disease, a high residual risk is observed in statin trials. This residual risk is partly explained by lipoprotein abnormalities other than LDL. Fenofibrate exerts a favorable effect on the atherogenic lipid profile of mixed dyslipidemia and can effectively reduce cardiovascular disease in patients with mixed dyslipidemia. Fenofibrate may offer important treatment alternatives as a second-line therapy in several circumstances: in combination with a statin for patients with mixed dyslipidemias not at goals on statin monotherapy; in monotherapy for patients intolerant or with contraindication to statin therapy; and in combination with other drugs (ezetimibe, colesevelam) for patients with mixed dyslipidemias, known intolerance, or contraindication to statin and not at goals on fenofibrate monotherapy. However, the role of fenofibrate-statin therapy and of other therapies involving fenofibrate in cardiovascular risk reduction strategies remains to be established.
\end{abstract}

Keywords: fenofibrate, mixed dyslipidemia, triglycerides, LDL-cholesterol, HDLcholesterol

Around the world, cardiovascular disease (CVD) is the leading cause of deaths for adults (World Health Statistics 2008). The most important modifiable cardiovascular risk factors are smoking, hypertension, dyslipidemia, obesity (mainly central) and hyperglycemia. These risk factors are often clustered, particularly for patients with type 2 diabetes and/or metabolic syndrome. Among dyslipoproteinemia, mixed or combined hyperlipidemia is a common disorder, occurring in about $30 \%$ of myocardial infarction (MI) survivors (Durrington 2003). Mixed dyslipidemia is characterized by elevated levels of triglycerides (TG) and reduced high-density lipoprotein cholesterol (HDL-C), with or without elevated levels of low-density lipoprotein cholesterol (LDL-C). This atherogenic lipoprotein phenotype is usually associated with a preponderance of small, dense LDL particles and an elevated apolipoprotein $\mathrm{B}(\mathrm{ApoB})$ concentration. All the metabolic abnormalities of mixed dyslipidemia contribute to increase risk for CVD. Mixed dyslipidemia can be genetically determined such as in familial combined hyperlipidemia and is also the must frequent lipid disorder found in patients with type 2 diabetes and metabolic syndrome (Best and O’Neal 2000; Szapary and Rader 2004). This review updates the management of mixed dyslipidemia and particularly on the clinical utility of fenofibrate in mixed dyslipidemia.

\section{Management of mixed dyslipidemia}

Mixed dyslipidemia is usually characterized by elevated LDL-C and TG and decreased HDL-C levels. Although plasma levels of LDL-C may be normal or only slightly 
above target levels, an increase in atherogenic small, dense LDL is a common feature, as well as elevated non-HDL-C and ApoB levels. Numerous landmark trials involving HMGCoA reductase inhibitors (statins) have firmly demonstrated that LDL-C lowering significantly reduces total mortality and CVD morbidity and mortality (CTT Collaborators 2005). Therefore, the use of statins has become the cornerstone of lipid lowering therapy in reducing the risk of CVD. Statins are indicated as first-line therapy for patients for primary hypercholesterolemia and mixed dyslipidemia. Moreover recent trials comparing different statin treatments have shown that intensive LDL-C lowering regimens are significantly more effective than moderate treatments in reducing coronary events and atherosclerotic progression (Nissen et al 2004; Cannon et al 2006).

Elevated LDL-C is identified as the primary target of lipid-lowering therapy by both US (NCEP ATP III 2001; Grundy et al 2004a) and European (European guidelines 2007; The Task Force on Diabetes 2007) guidelines. These guidelines specified LDL-C of $100 \mathrm{mg} / \mathrm{dL}(2.6 \mathrm{mmol} / \mathrm{L})$ as a minimal goal of treatment in high risk patients. For patients at very high risk, a new therapeutic option for LDL-C is below 70 to $80 \mathrm{mg} / \mathrm{dL}(<2 \mathrm{mmol} / \mathrm{L})$. The National Cholesterol Education Program Adult Treatment Panel III (NCEP ATP III) recognized both low HDL-C $(<40 \mathrm{mg} / \mathrm{dL})$ and elevated $\mathrm{TG}(\geq 150 \mathrm{mg} / \mathrm{dL})$ as markers of increased CVD risk. Beyond lowering LDL-C, the NCEP ATP III introduced a secondary target of therapy, non-HDL-C, in patients with elevated TG. In some studies, non-HDL-C has been reported to be a stronger predictor of CVD risk than LDL-C (Cui et al 2001; Ridker et al 2005). More recently, in a post hoc analysis of TNT and IDEAL trials (Kastelein et al 2008), on-statin treatment levels of non-HDL-C and apolipoprotein B were more closely associated with cardiovascular outcome than LDL-C levels, supporting the use of non-HDL-C as a novel treatment target. The goal for non-HDL-C at any level of risk is $30 \mathrm{mg} / \mathrm{dL}$ higher than the LDL-C treatment goal. Thus, in patients with mixed dyslipidemia and very high risk (ie, with CVD and diabetes or the metabolic syndrome (Grundy et al 2004a) who have an LDL-C goal of $70 \mathrm{mg} / \mathrm{dL}$, the non-HDL-C goal is $100 \mathrm{mg} / \mathrm{dL}$. The coronary risk is also associated with mildly elevated (150-500 mg/dL) plasma TG level. Very high levels of TG $(>500 \mathrm{mg} / \mathrm{dL})$ are a risk for acute pancreatitis.

In high risk patients with mixed dyslipidemia, an ideal treatment should deal with the global spectrum of the atherogenic lipid triad (high LDL-C, low HDL-C and high TG). High levels of LDL-C could be effectively controlled with a statin. However statin therapy may be limited by the failure to reach non-HDL-C goals and sometimes by intolerance or poor response in monotherapy. Several considerations have led to increased interest in non-statin options for correction of mixed dyslipidemia:

- Low HDL-C is associated with increased CVD risk (Gordon et al 1977, 1989). The Framingham study indicated a clear relationship between low HDL-C levels and increased risk of coronary disease, irrespective of LDL-C levels (Gordon et al 1977). In a meta-analysis of four large prospective studies (Gordon et al 1989), every $1 \mathrm{mg} / \mathrm{dL}$ decrease in HDL-C is associated with increased risk of coronary heart disease (CHD), differently in males and females ( $2 \%$ males and $3 \%$ females), independent of other risk factors, including LDL-C levels.

- Even if TG have been a controversial risk factor, recent epidemiologic evidences have demonstrated that elevated TG independently predicts CVD. A recent meta-analysis of 29 prospective studies enrolling 262,525 subjects indicated a strong and highly significant association between TG values and CHD risk (Sarwar et al 2007). Two additional studies have corroborated these findings (Bansal et al 2007; Nordestgaard et al 2007), both studies supporting the concept that non-fasting TG levels may strongly predicts the CVD risk (McBride 2007). In mixed dyslipidemia, post-prandial triglyceride-rich lipoproteins are typically increased with a delay in the clearance of these lipoprotein particles and a prolonged exposure.

- Although statins are clearly effective in lowering LDL-C levels, this class of drugs appear to have more modest effects in terms of raising HDL-C and lowering TG. Statins have been reported to raise HDL-C levels by $5 \%$ to $15 \%$ and to lower TG by $7 \%$ to $30 \%$ (NCEP ATP III 2001).

- Finally in landmark statin clinical trials, high "residual" CVD risk can be partly explained by the presence of lipoprotein abnormalities other than LDL. Indeed, subjects treated with a statin but who had low HDL-C levels remained at a greater CVD risk than those with normal or high HDL-C levels. In a recent post-hoc analysis of the Treating to New Targets (TNT) trial, levels of HDL-C were inversely related to the risk of major CVD events among CHD patients receiving statin treatment (Barter et al 2007). Even in patients with LDL-C at or below the optional treatment target of $70 \mathrm{mg} / \mathrm{dL}$, those who were in the lowest HDL-C quintile had a significantly increased CVD risk compared with those in the highest HDL-C quintile (Barter et al 2007). Interestingly 
a similar finding has been found for TG levels in a complementary analysis of PROVE-IT TIMI-22 trial (Miller et al 2008): compared with individuals who achieved low levels of both LDL-C $(<70 \mathrm{mg} / \mathrm{dL})$ and TG $(<150 \mathrm{mg} / \mathrm{dL})$, patients with higher levels of ontreatment LDL-C and/or TG had significantly elevated risk of CHD events. On-treatment TG $<150 \mathrm{mg} / \mathrm{dL}$ was independently associated with a lower risk of recurrent CHD effects.

Conversely, a number of studies have demonstrated that treatment with fibrates or niacin (with or without a statin) improves the atherogenic lipoprotein profile and/or reduces cardiovascular morbidity and mortality. Among these drugs, this review only reports arguments for a clinical utility of fenofibrate.

\section{Mechanism of action of fenofibrate}

Fenofibrate belongs to a class of drugs that exert their effects by activating the peroxisome proliferator-activated receptor $\alpha$ $(\operatorname{PPAR} \alpha)$, a transcription factor that regulates lipid metabolism via a number of routes. Activated PPAR $\alpha$ stimulates the expression of genes encoding various enzymes that regulate fatty acid and lipoprotein metabolism (Fruchart et al 2001; Fazio and Linton 2004; Keating and Croom 2007). Fenofibrate stimulated the oxidation of free fatty acids in the liver. This promotion of the $\beta$-oxidation of fatty acids reduced the availability of fatty acids for very-low density lipoprotein (VLDL) synthesis and secretion. Fenofibrate also increased the expression of the gene for lipoprotein lipase and decreases ApoC-III expression in the liver. Thus, fenofibrate lowered the concentration of TG both by reducing the rate of synthesis and increasing the rate of hydrolysis of triglyceride-rich lipoproteins (Staels et al 1998; Keating and Croom 2007). Moreover, fenofibrate treatment reduced the proportion of small, dense LDL, with the formation of larger, less dense LDL particles with a higher affinity for the LDL receptor and thus catabolized more rapidly (Chapman 2006). PPAR $\alpha$ activation with fenofibrate also increased expression of the genes for both ApoA-I and ApoA-II, decreased the cholesteryl ester transfer protein-mediated transfer of cholesterol from HDL to VLDL, enhanced cell cholesterol efflux by induction of cell ABCA1 expression and decreased SR-B1 in the liver. All these effects contribute to the increase of plasma HDL-C concentrations (Guerin et al 1996; Chinetti et al 2001; Mardones et al 2003; Fruchart and Duriez 2006).

In addition to the lipid-modifying activity, fenofibrate also had numerous pleiotropic effects mediated by PPAR $\alpha$ activation (Staels et al 1998; Paumelle and Staels 2008): fenofibrate improved endothelial function (Playford et al 2002; Capell et al 2003; Koh et al 2005) and exerted anti-inflammatory activities (Zambon et al 2006; Rosenson et al 2008) as evidenced by a reduction in CRP as well as a number of cytokines (eg, IL-6, TNF-alpha) In patients with metabolic syndrome and elevated TG, fenofibrate reduced whole blood production of inflammatory cytokines and hepaticsynthesized inflammatory proteins, and the anti-inflammatory effects of fenofibrate involve VLDL- and LDL-mediated pathways (Rosenson et al 2008). Fenofibrate also decreased procoagulant factors such as fibrinogen and plasminogen activator inhibitor-1 (Kaneko et al 2002; Maison et al 2002) and reduced monocyte chemoattractant protein-1 (Keating and Ormrod 2002; Paumelle and Staels 2008). Among fibrates, only fenofibrate significantly reduced uric acid levels (Liamis et al 1999; Keating and Ormrod 2002).

In contrast, fenofibrate therapy induced two potential deleterious effects: an increase in creatinine (Keating and Ormrod 2002; Tsimichodimos et al 2002) and homocysteine levels (Keating and Ormrod 2002; Dierkes et al 2003). Whether these effects on creatinine and homocysteine are of clinical importance remains to be determined. Creatinine rises was not associated with an alteration in renal function, as measured by glomerular filtration rate (Hottelart et al 2002). The creatinine elevation associated with fenofibrate treatment is fully reversible within a few weeks of ceasing therapy (The FIELD study investigators 2005), also suggesting an absence of permanent renal damage.

The potential of monotherapy with fenofibrate in the treatment of patients with primary dyslipidemia has been well established in numerous, placebo-controlled and comparative trials (Adkins and Faulds 1997; Keating and Ormrod 2002). The effect of fenofibrate on the concentration of plasma lipids is largely influenced by the baseline lipid levels. Fenofibrate therapy was consistently associated with a substantial decreases of serum TG by $20 \%$ to $50 \%$, usually directly proportional to the baseline TG levels. Fenofibrate increased HDL-C levels by $10 \%$ to $25 \%$, to a degree directly dependent of baseline TG and HDL-C levels. Fenofibrate had widely variable effects on LDL-C levels mainly dependent of the type of dylipidemia. For example, fenofibrate may decrease LDL-C by $20 \%$ in patients with normal TG levels, but generally produces less reduction in LDL-C in patients with mixed dyslipidemia. Patients with severe hypertriglyceridemia and low levels of LDL-C may raise their LDL-C levels during fenofibrate treatment, possibly as a result of an accelerated catabolism of triglyceride-rich lipoproteins, leading to an increased LDL conversion and an increased LDL particle size. This increase in LDL size may constitute an antiatherogenic mechanism. 


\section{Clinical utility of fenofibrate in mixed dyslipidemia Clinical efficacy in monotherapy}

A meta-analysis of randomized fibrate trials (1,457 patients receiving fenofibrate) realized across a range of populations, found that fenofibrate decreased LDL-C and TG respectively by $11 \%$ and $40 \%$ and increased HDL-C by $10 \%$ (Birjmohun et al 2005).

Although the effect of fenofibrate on LDL-C in these studies was variable, it was generally smaller than that achieved with statins. Two recent trials (Farnier et al 2005; Farnier et al 2007) provided data on the efficacy of fenofibrate monotherapy in large populations of patients with mixed dyslipidemia: in these trials, TG decreased by $43.2 \%$ and $41.3 \%$, HDL-C increased by $18.8 \%$ and $18.2 \%$, LDL-C decreased by $5.5 \%$ and $15.7 \%$, and non-HDL-C decreased by $16.2 \%$ and $21.0 \%$ (Farnier et al 2005; Farnier et al 2007). The effects on ApoB were in agreement with the evolution of non-HDL-C levels: ApoB levels decreased by $15.2 \%$ and $20.1 \%$. ApoA-I levels were increased by $8.4 \%$ and $10.8 \%$ (Farnier et al 2005; Farnier et al 2007). Finally these trials have confirmed a large and significant shift in LDL size with a decrease in the proportion of small, dense LDL during fenofibrate treatment. In summary, fenofibrate has a favorable action on all the lipoprotein abnormalities present in patients with mixed dyslipidemia.

This pattern of mixed dyslipidemia with high TG, low HDL-C and preponderance of small dense LDL is usually observed in type 2 diabetes and metabolic syndrome. Presumably to follow-up on the favorable effects of gemfibrozil treated diabetic patients in Helsinki Heart Study (HHS) and Veterans Affairs High-Density Lipoprotein Cholesterol Intervention Trial (VA-HIT) study, the potential role of fenofibrate for CVD risk reduction has only been evaluated in type 2 diabetes, but not specifically type 2 diabetic patients with mixed dyslipidemia.

In the Diabetes Atherosclerosis Intervention Study (DAIS 2001), 418 diabetic men and women were randomized to fenofibrate or placebo for 3 years. Baseline lipids were LDL-C of $132 \mathrm{mg} / \mathrm{dL}$, TG of $221 \mathrm{mg} / \mathrm{dL}$, and HDL-C of $40 \mathrm{mg} / \mathrm{dL}$. Fenofibrate lowered LDL-C by $6 \%$ and TG by $28 \%$, and raised HDL-C by $7 \%$. Fenofibrate slowed the angiographic progression of coronary atherosclerosis: the progression of focal coronary atheroma was $40 \%$ less in the fenofibrate group compared with placebo, without significant effect on diffuse atheroma. Interestingly, although the study was not powered to look at clinical events, there was a non significant $23 \%$ reduction in CVD events. These effects seemed to be explained not only by the changes in HDL-C, LDL-C and TG levels, but also by a significant increase in LDL particle size (Vakkilainen et al 2003). Additionally, fenofibrate reduced the incidence of microalbuminuria by 54\% (Ansquer et al 2005). In DAIS, the increase of homocysteine did not alter the beneficial effect of fenofibrate (Genest et al 2004).

The Fenofibrate Intervention and Event Lowering in Diabetes (FIELD) was the first large landmark cardiovascular event-based trial with fenofibrate (The FIELD study investigators 2005). FIELD was a 5 -year, randomized, placebocontrolled, double-blind study of the efficacy and safety of fenofibrate $200 \mathrm{mg} /$ day in 9795 type 2 diabetic patients with (22\%) or without (78\%) previous CVD. Mean baseline lipids were LDL-C of $119 \mathrm{mg} / \mathrm{dL}$, TG of $153 \mathrm{mg} / \mathrm{dL}$, and HDL-C of $43 \mathrm{mg} / \mathrm{dL}$. Unfortunately, only $21 \%$ of patients had a marked dyslipidemia defined (for comparison with other fibrates trials) by $\mathrm{TG} \geq 200 \mathrm{mg} / \mathrm{dL}$ and low HDL-C $<40 \mathrm{mg} / \mathrm{dL}$ in men and $<50 \mathrm{mg} / \mathrm{dL}$ in women (Scott et al 2007). At 4 months and study close, fenofibrate decreased respectively TG by $29 \%$ and $22 \%$, LDL-C by $12 \%$ and $6 \%$ and increased HDL-C by $5 \%$ and $1 \%$. Fenofibrate treatment did not have a significant effect on the primary end point (CHD death or non-fatal MI) reduced by only $11 \%(p=0.16)$. There was, however, a significant reduction $(-11 \%, p=0.035)$ in the secondary end point of total CVD events (a composite of CVD death, MI, stroke, and coronary or carotid revascularization), largely driven by significant reductions in non-fatal MI $(-24 \%$, $\mathrm{p}=0.01)$ and coronary revascularization $(-21 \%, \mathrm{p}=0.003)$. There was a non-significant increase in CHD death in the fenofibrate group. The FIELD investigators also reported a significant $20 \%$ reduction of all MI events $(p=0.006)$ and a significant $38 \%$ reduction in the risk of non-traumatic amputations ( $p=0.011)$ with fenofibrate (Burgess et al 2007). Fenofibrate treatment was also associated with significant reductions in the need for retinal laser therapy and decreased progression/increased regression of albuminuria (The FIELD study investigators 2005). The global results of FIELD have been considered as disappointing event if the overall CVD event results were positive in several regards.

Some explanations can be proposed to explain the FIELD results: the higher rate of statin use in the placebo group (36\% among placebo patients by close of study versus $19 \%$ in the fenofibrate arm) may play a role. Among fenofibrate patients not receiving off-trial lipid treatment, the CVD composite end point was significantly reduced by $19 \%$. The poor effect of fenofibrate in reducing CVD events, more particularly in secondary prevention, could also be explained by the significant increase 
of homocysteine levels. It has been shown that gemfibrozil increased plasma homocysteine less than fenofibrate (Dierkes et al 2003; Syvanne et al 2004) and this difference could explain the better clinical benefit of gemfibrozil in the VA-HIT (Robins et al 2001; Rubins et al 2002) and HHS (Frick et al 1987). Another hypothesis to explain the FIELD results is the modest effects of fenofibrate on lipid parameters; particularly the declining effect on HDL-C overtime could also be due to the fact to elevated homocysteine has been reported to reduce the ApoA-I expression (Mikael et al 2006).

More convincing, the relative lack of favorable effects in FIELD may be related to the selected population. Fibrates reduce CVD effectively in patients with insulin resistance/ overweight people with high TG and low HDL-C (Barter and Rye 2008). In FIELD, the baseline TG was lower and the HDL-C was higher than in HHS or VA-HIT, with a lower TG/HDL-C ratio (4.05) than in VA-HIT (higher ratio of 5.37). New complementary analyses recently reported (Scott et al 2007) have provided important data on the clinical utility of fenofibrate in mixed dyslipidemia: in the subgroup of 2,014 patients with low HDL-C and TG $>200 \mathrm{mg} / \mathrm{dL}$, fenofibrate treatment induced a significant reduction of CVD events $(13.5 \%$ in the fenofibrate group versus $17.8 \%$ in the placebo group, HR $0.74, \mathrm{p}=0.007$ ). This highly significant effect corresponds to a $4.3 \%$ absolute risk reduction, with a number need to treat of 23 to avoid 1 or more CVD events. The beneficial effect of fenofibrate on macrovascular events appears larger among type 2 diabetic subjects with mixed dyslipidemia (Scott et al 2007).

Fenofibrate was generally well tolerated. A meta-analysis of 53 trials using fibrates has not shown any increase in non coronary death or cancer (Birjmohun et al 2005). The most frequent adverse effects were gastrointestinal symptoms, skin reactions and musculoskeletal symptoms (Birjmohun et al 2005). Some cases of liver function test and creatine phosphokinase abnormalities have been reported (Keating and Ormrod 2002; Keating and Croom 2007). In the FIELD trial, it has been also observed a slight but significant increase in pancreatitis $(0.8 \%$ in fenofibrate group versus $0.5 \%$ in placebo group) and pulmonary embolism (1.1\% versus $0.7 \%$ ), and a non significant increase in deep vein thrombosis (1.4\% versus $1.0 \%$ ). The excess of pancreatitis may be due to the increased lithogenicity of bile. The increased risk of venous thrombotic events may be related to the increased homocysteine level, a risk factor for thrombosis (Undas et al 2005). Overall, in FIELD, rhabdomyolysis only occurred in three fenofibrate recipients and one placebo recipient, and all cases were fully resolved.
Fenofibrate is contraindicated in patients with hepatic or several renal dysfunction, pre-existing gallbladder disease, primary biliary cirrhosis or unexplained persistent liver function abnormalities. Fenofibrate potentiates the anticoagulant activity of coumarin and there is a warning on the concomitant use of fenofibrate and cyclosporine.

\section{Clinical efficacy in combination with statins}

Guidelines suggest both fibrates and niacin as additions to statin therapy in high risk patients with persistently low levels of HDL-C and elevated triglycerides (Grundy et al 2004a; Buse et al 2007; The Task Force on Diabetes 2007). Updated guidelines from the NCEP ATP III recognize the potential of the statin-fibrate and statin-niacin combination therapies in patients with mixed dyslipidemia and CHD or CHD risk equivalents (Stone et al 2005).

Given the complementary pharmacologic profiles of fibrates and statins, greater clinical benefit may be expected with combination therapy. However the use of fibrate/statin combination therapy has been rapidly restricted due to safety concerns, mainly an increased risk of myopathy and rhabdomyolysis (Shek and Ferrill 2001; Farnier 2003). Several reviews have examined the beneficial effects and the safety of a statin-fibrate combination therapy (Shek and Ferrill 2001; Farnier 2003; Wierzbicki et al 2003; Stefanutti et al 2004; Corsini et al 2005). In summary, the available evidence on safety clearly indicates differences in the risk of myopathy and rhabdomyolysis between fibrates (Alsheikh-Ali et al 2004; Jones and Davidson 2005). In combination with a statin, rhabdomyolysis has been reported to be 15 -fold (Jones and Davidson 2005) and 33-fold (Alsheikh-Ali et al 2004) higher with gemfibrozil than with fenofibrate. Moreover the risk was particularly elevated when cerivastatin was use in combination with gemfibrozil (Chang et al 2004; Graham et al 2004; Jones and Davidson 2005). The pharmacokinetic interactions between statins and fibrates have been carefully studied (Prueksaritanont et al 2002a; Corsini et al 2005). The mechanism for the higher rate of adverse interaction of gemfibrozil versus fenofibrate with statins appears mainly to be interference with statin glucuronidation by gemfibrozil. In vitro studies have demonstrated that gemfibrozil interacts with the same family of glucuronidation enzymes that are involved in statin metabolism (Prueksaritanont et al 2002a, b). In contrast, fenofibrate was metabolized by different glucuronidation enzymes than those involved in statin metabolism (Prueksaritanont et al 2002b). Therefore, gemfibrozil causes a 2- to 6-fold increase in the statin AUC 
(Corsini et al 2005). But fenofibrate has not significant effect on the pharmacokinetics of various statins (Pan et al 2000; Martin et al 2003; Bergman et al 2004, Gustavson et al 2005). Finally, clinical trial data have not reported an increased risk of myopathy with various statins combined to fenofibrate (Ellen and McPherson 1998; Farnier and Dejager 2000; Athyros et al 2002; Martin et al 2003; Vega et al 2003; Durrington et al 2004; Grundy et al 2005). In FIELD, among the 944 patients taking fenofibrate and statin combination therapy in about 2000 patient-years of use, no cases of rhabdomyolysis were reported (The FIELD study investigators 2005; Keech 2006).

All these data suggest that fenofibrate may be the preferred fibrate for use in combination with statin and guidelines recommend fenofibrate as the fibrate of choice for high-risk statin-treated patients with mixed dyslipidemia (Grundy et al 2004b). Fenofibrate may be added to a statin when mixed dyslipidemia responds inadequately with monotherapy, although use of lower statin doses has been recommended in this case (Davidson et al 2007).

The results of clinical studies support the efficacy of fenofibrate-statin therapy in patients with mixed dyslipidemia (Ellen and McPherson 1998; Athyros et al 2002; Vega et al 2003; Durrington et al 2004; Grundy et al 2005). For example, in the SAFARI trial (Grundy et al 2005), 619 patients with mixed dyslipidemia (TG from 150 to 500 $\mathrm{mg} / \mathrm{dL}$ and LDL-C higher than $130 \mathrm{mg} / \mathrm{dL}$ ) were treated with either simvastatin $20 \mathrm{mg} / \mathrm{d}$ or the combination of simvastatin $20 \mathrm{mg} / \mathrm{d}$ plus fenofibrate $160 \mathrm{mg} / \mathrm{d}$ for 12 weeks. The combined treatment was associated with significantly greater changes compared with simvastatin alone in TG $(-43.0 \%$ versus $-20.1 \%)$, LDL-C $(-31.2 \%$ versus $-25.8 \%)$, non-HDL-C $(-35.3 \%$ versus $-26.1 \%)$, and HDL-C $(+18.6 \%$ versus $+9.7 \%)$. In addition, combination therapy induced a significant shift from small, dense LDL particles to larger, more buoyant LDL particles (Grundy et al 2005).

The same beneficial effect on LDL subfractions has been observed in the DIACOR study in the group of patients with type 2 diabetes and mixed dyslipidemia receiving the combination of simvastatin $20 \mathrm{mg} / \mathrm{d}$ and fenofibrate $160 \mathrm{mg} / \mathrm{d}$ (May et al 2008). The DIACOR trial has also shown that combination therapy has greater anti-inflammatory effects than either form of monotherapy (Muhlestein et al 2006), probably in relation with the cross-talk between statins and PPAR $\alpha$ agonists regarding pleiotropic effects (Paumelle and Staels 2008). Several trials are ongoing to evaluate more precisely the complementary beneficial effects of fenofibrate for patients with mixed dyslipidemia not at goals on statin therapy.
All these data may support the use of fenofibrate-statin combination therapy to achieve a global lipid and vascular control and suggest that atherosclerosis and CVD benefits may be greater with combination therapy, but direct evidence of these benefits is not yet available. Hopefully, the ongoing Action to Control Cardiovascular Risk in Diabetes (ACCORD) study was designed to evaluate whether adding fenofibrate to simvastatin can reduce CVD risk beyond the risk reduction with simvastatin alone in type 2 diabetic patients (The ACCORD Study Group 2007; Ginsberg et al 2007).

\section{Clinical efficacy in combination therapy with other agents}

Although statins are the drug of first choice in patients with mixed dyslipidemia, the use of statins may be limited by intolerance or poor response in monotherapy.

In patients who are unable to tolerate statin therapy, two alternative combination therapies with fenofibrate can be proposed at patients with mixed dyslipidemia, either fenofibrate-ezetimibe or fenofibrate-colesevelam. The efficacy and safety of fenofibrate plus ezetimibe combination therapy has been evaluated in 625 patients with mixed dyslipidemia [TG from 200 to $500 \mathrm{mg} / \mathrm{dL}$, LDL-C from 130 to $220 \mathrm{mg} / \mathrm{dL}$ $(100-180 \mathrm{mg} / \mathrm{dL}$ in patients with diabetes)] (Farnier et al 2005; McKenney et al 2006) randomized to received one of the four daily treatments: placebo, ezetimibe $10 \mathrm{mg}$, fenofibrate $160 \mathrm{mg}$ and fenofibrate $160 \mathrm{mg}$ + ezetimibe $10 \mathrm{mg}$ during 12 weeks. The complementary effects of fenofibrate and ezetimibe improve the overall atherogenic lipid profile, with decreases in LDL-C of 20.4\%, non-HDL-C of 30.4\%, TG of $44.0 \%$, and increase in HDL-C of $19.0 \%$ (Farnier et al 2005). Moreover, the co-administration of fenofibrate and ezetimibe produced complementary and favorable changes in lipoprotein subfractions, promoting a shift in the LDL particle distribution profile toward larger, more buoyant particles (Farnier et al 2005; Tribble et al 2008). After completing the 12-week, randomized, double-blind base study, 576 patients entered in a 48-week, double-blind extension study, during which they received fenofibrate or fenofibrate plus ezetimibe (McKenney et al 2006). Improvements from baseline in LDL-C (-22.0\% versus $-8.6 \%)$, non-HDL-C $(-31.6 \%$ versus $-19.4 \%)$, TG $(-46.0 \%$ versus $-41.8 \%)$ and HDL-C (20.9\% versus $17.8 \%)$ levels were significantly greater with fenofibrate-ezetimibe combination therapy than with fenofibrate alone. The combination of ezetimibe plus fenofibrate was well tolerated during both the base study (Farnier et al 2005) and the extension study (McKenney et al 2006). In the base study, one patient receiving combination therapy 
was discontinued after being diagnosed with cholelithiasis and subsequent cholecystectomy. In the extension study, the proportion of patients with cholecystectomy was not significantly different between treatments, but this study was not designed to assess this infrequent biliary adverse event. It has been reported that fenofibrate may increase cholesterol excretion into bile and ezetimibe has inconsistent effects on biliary cholesterol in animal models (Farnier 2007).

Another alternative to statin therapy is a bile acid sequestrant (BAS) combined with a fibrate. Colesevelam has become the preferred BAS because it is well-tolerated compared to older BAS and lowers LDL-C levels an average of $15 \%$ to $18 \%$. The complementary efficacy of colesevelam added to fenofibrate has been evaluated in 129 patients with mixed dyslipidemia treated 8 weeks by fenofibrate $160 \mathrm{mg} / \mathrm{d}$, then randomized to receive either colesevelam $3.75 \mathrm{~g} / \mathrm{d}$ or placebo (McKenney et al 2005). Compared with fenofibrate monotherapy, the combination of fenofibrate and colesevelam significantly reduced LDL-C (-17.0\%) and non-HDL-C (-21.0\%) from baseline, without affecting the TG-lowering or HDL-C raising effects of fenofibrate (McKenney et al 2005).

Finally in patients with severe mixed dyslipidemia or poor responders to statin monotherapy, a new option is a triple therapy using statin, ezetimibe and fenofibrate. The efficacy of the co-administration of ezetimibe/simvastatin $10 / 20 \mathrm{mg}+$ fenofibrate $160 \mathrm{mg}$ has been evaluated in 611 patients with mixed dyslipidemia (TG from 150 to $500 \mathrm{mg} / \mathrm{dL}$, LDL-C from 130 to $220 \mathrm{mg} / \mathrm{dL}$ [100-180 $\mathrm{mg} / \mathrm{dL}$ in patients with diabetes]) randomized to receive one of the four treatments ezetimibe/simvastatin $10 / 20 \mathrm{mg}+$ fenofibrate $160 \mathrm{mg}$, ezetimibe $10 / 20 \mathrm{mg}$, fenofibrate $160 \mathrm{mg}$, or placebo for 12 weeks (Farnier et al 2007). The triple therapy improved the overall atherogenic lipid profile ( $-46 \%$ for LDL-C, $-50 \%$ for TG and non-HDL-C, $-45 \%$ for ApoB, $+19 \%$ for HDL-C) with a significant shift of small, dense LDL to larger particles (Farnier et al 2007). Although the co-administration of fenofibrate and ezetimibe/simvastatin was well tolerated in this short-term study, the long-term safety and clinical outcome benefits remain to be determined.

\section{Rational prescribing of fenofibrate in mixed dyslipidemias}

Although statins are indicated as first-line therapy for patients with mixed dyslipidemia, statin treatment may be limited by the failure to reach LDL-C and non-HDL-C targets and by intolerance or poor-response in monotherapy. Moreover, many patients remain at risk of CVD despite having LDL-C levels below recommended targets. Thus, increasing attention is being focused on other lipoprotein fractions such as HDL and triglycerides, as potential targets of therapy. There is recent evidence that abnormalities of the triglyceride-HDL axis are especially associated with adverse cardiovascular outcomes. In FIELD, fenofibrate reduced CVD effectively in patients with insulin resistance, low HDL-C, and high TG. However, prescribing recommendations are constrained by a lack of clinical trial evidence of fenofibrate-statin therapy. Nevertheless, it is possible to propose a rational prescribing of fenofibrate in mixed dyslipidemia:

1. For high-risk patients not at their non-HDL-C goals and with high TG and/or low HDL-C levels, a statinfenofibrate combination therapy can be required to control all lipid abnormalities. In light of the increasing prevalence of diabetes and the metabolic syndrome, the clinical use of fenofibrate combined with a statin may be likely to increase. Data from prospective outcome studies are required to evaluate the benefits of this approach.

In severe mixed dyslipidemia, when the LDL-C and non-HDL-C goals are not reached with a usual dose of statin and fenofibrate, a triple therapy with a statin, fenofibrate, and ezetimibe can be used to avoid the highest statin doses and with the same caution as statinfenofibrate therapy.

2. For patients with intolerance to statin therapy or if a statin is inappropriate or contraindicated, fenofibrate monotherapy is indicated in mixed dyslipidemia. However, particularly for high risk patients, the LDL$\mathrm{C}$ and/or non-HDL-C goals are often not attained with fenofibrate alone. A combination of fenofibrate wth ezetimibe or colesevelam can be useful for these patients.

In conclusion, despite the impressive benefits achieved by LDL-C lowering using statins, there are compelling reasons to consider other lipoprotein abnormalities present in mixed dyslipidemia as risk factors. Fenofibrate has a favorable effect on the global atherogenic lipoprotein profile and may offer important treatment alternatives as second-line therapy.

\section{Disclosures}

The author reports having received grant/research support and/or honoraria from a consultant for Astra-Zeneca, Genfit, 
Genzyme, Kowa, Merck and Co, Merck-Schering-Plough, Novartis, Pfizer, Roche, Sanofi-Aventis, Solvay, and Takeda.

\section{References}

Adkins JC, Faulds D. 1997. Micronised fenofibrate. A review of its pharmacodynamic properties and clinical efficacy in the management of dyslipidaemia. Drugs, 54:615-33.

Alsheikh-Ali AA, Kuvin JT, Karas RH. 2004. Risk of adverse events with fibrates. Am J Cardiol, 94:935-8.

Ansquer JC, Foucher C, Rattier S, et al. 2005. Fenofibrate reduces progression to microalbuminuria over 3 years in a placebo-controlled study in type 2 diabetes: results from the Diabetes Atherosclerosis Intervention Study (DAIS). Am J Kidney Dis, 45:485-93.

Athyros VG, Papageorgiou AA, Athyrou VV, et al. 2002. Atorvastatin and micronized fenofibrate alone and in combination in type 2 diabetes with combined hyperlipidemia. Diabetes Care, 25:1198-202.

Bansal S, Buring JE, Rifai N, et al. 2007. Fasting compared with nonfasting triglycerides and risk of cardiovascular events in women. JAMA, 298:309-16.

Barter P, Gotto AM, La Rosa JC, et al. 2007. HDL cholesterol, very low levels of LDL cholesterol, and cardiovascular events. $N$ Engl J Med, 357:1301-10.

Barter PJ, Rye KA. 2008. Is there a role for fibrates in the management of dyslipidemia in the metabolic syndrome? Arterioscler Thromb Vasc Biol, 28:39-46.

Bergman AJ, Murphy G, Burke J, et al. 2004. Simvastatin does not have a clinically significant pharmacokinetic interaction with fenofibrate in humans. J Clin Pharmacol, 44:1054-62.

Best JD, O’Neal DN. 2000. Diabetic dyslipidemia. Current treatment recommendations. Drugs, 59:1101-11.

Birjmohun RS, Hutten BA, Kastelein JJP, et al. 2005. Efficacy and safety of high-density lipoprotein cholesterol-increasing coumpounds: a meta-analysis of randomized controlled trials. J Am Coll Cardiol, 45:185-97.

Burgess D, Hunt D, Li L, et al. 2007. on behalf of the FIELD Investigators. Effects of fenofibrate on silent myocardial infarction, hospitalization for acute coronary syndromes and amputation in type 2 diabetes: the Fenofibrate Intervention and Event Lowering in Diabetes (FIELD) study. Circulation, 116(Suppl II):838.

Buse JB, Ginsberg HN, Bakris GL, et al. 2007. for the American Heart Association and the American Diabetes Association. Primary prevention of cardiovascular diseases in people with diabetes mellitus: a scientific statement from the American Heart Association and the American Diabetes Association. Circulation, 115:114-26.

Cannon CP, Steinberg BA, Murphy SA, et al. 2006. Meta-analysis of cardiovascular outcomes trials comparing intensive versus moderate statin therapy. J Am Coll Cardiol, 48:438-45.

Capell WH, De Souza CA, Poirier P, et al. 2003. Short-term triglyceride lowering with fenofibrate improves vasodilator function in subjects with hypertriglyceridemia. Arterioscler Thromb Vasc Biol, 23:307-13.

Chang JT, Staffa JA, Parks M, et al. 2004. Rhabdomyolysis with HMGCoA reductase inhibitors and gemfibrozil combination therapy. Pharmacoepidemiol Drug Safety, 13:417-26.

Chapman MJ. 2006. Fibrates: therapeutic review. Br J Diabetes Vasc Dis, $6: 11-8$.

Chinetti G, Lestavel S, Bocher V, et al. 2001. PPAR-alpha and PPARgamma activators induce cholesterol removal from human macrophage foam cells through stimulation of the ABCA1 pathway. Nat Med, 7:53-8.

Cholesterol Treatment Trialist's (CTT) Collaborators. 2005. Efficacy and safety of cholesterol-lowering treatment: prospective meta-analysis of data from 90056 participants in 14 randomised trials of statins. Lancet, 366:1267-78.
Corsini A, Bellosta S, Davidson MH. 2005. Pharmacokinetic interactions between statins and fibrates. Am J Cardiol, 96(Suppl):44K-49K.

Cui Y, Blumenthal RS, Flaws JA, et al. 2001. Non-high-density lipoprotein cholesterol level as a predictor of cardiovascular disease mortality. Arch Intern Med, 161:1413-9.

Davidson MH, Armani A, McKenney JM, et al. 2007. Safety considerations with fibrate therapy. Am J Cardiol, 99:3C-18C.

[DAIS] Diabetes Atherosclerosis Intervention Study Investigators. 2001. Effect of fenofibrate on progression of coronary-artery disease in type 2 diabetes: the Diabetes Atherosclerosis Intervention Study. Lancet, 357:905-10.

Dierkes J, Westphal S, Luley C. 2003. Fenofibrate-induced hyperhomocysteinaemia. Clinical implications and management. Drug Safety, 26:81-91

Durrington, P. 2003. Dyslipidaemia. Lancet, 362:717-31.

Durrington PN, Tuomiletho J, Hamann A, et al. 2004. Rosuvastatin and fenofibrate alone and in combination in type 2 diabetes patients with combined hyperlipidaemia. Diab Res Clin Pract, 64:137-51.

Ellen RLB, McPherson R. 1998. Long-term efficacy and safety of fenofibrate and a statin in the treatment of combined hyperlipidemia. $A m J$ Cardiol, 81(4A):60B-65B.

European guidelines on cardiovascular disease prevention in clinical practice: full text. 2007. Fourth Joint Task Force of the European Society of Cardiology and other societies on cardiovascular disease prevention in clinical practice. Eur J Cardiovasc Prev Rehabil, 14(Suppl 2): S1-S113.

Farnier M. 2003. Combination therapy with an HMG-CoA reductase inhibitor and a fibric acid derivative. A critical review of potential benefits and drawbacks. Am J Cardiovasc Drugs, 3:169-78.

Farnier M. 2007. Ezetimibe plus fenofibrate: a new combination therapy for the management of mixed hyperlipidaemia? Expert Opin Pharmacother, 8:1345-52.

Farnier M, Dejager S; and the French Fluvastatin Study Group. 2000. Effect of combined fluvastatin-fenofibrate therapy compared with fenofibrate monotherapy in severe primary hypercholesterolemia. $\mathrm{Am}$ J Cardiol, 85:53-7.

Farnier M, Freeman MW, Macdonnel G, et al. 2005. Efficacy and safety of the coadministration of ezetimibe with fenofibrate in patients with mixed hyperlipidaemia. Eur Heart J, 26:897-905.

Farnier M, Roth E, Gil-Extremera B, et al; for the Ezetimibe/Simvastatin + Fenofibrate Study Group. 2007. Efficacy and safety of the coadministration of ezetimibe/simvastatin with fenofibrate in patients with mixed hyperlipidemia. Am Heart $J$, 153:335e1-335e8.

Fazio S, Linton McRF. 2004. The role of fibrates in managing hyperlipidemia: mechanisms of action and clinical efficacy. Curr Atheroscl Rep, 6:148-57.

Frick MH, Elo O, Haapa K, et al. 1987. Helsinki Heart Study: primaryprevention trial with gemfibrozil in middle-aged men with dyslipidemia. Safety of treatment, changes in risk factors, and incidence of coronary heart disease. N Engl J Med, 317:1237-45.

Fruchart JC, Duriez P. 2006. Mode of action of fibrates in the regulation of triglycerides and HDL-cholesterol metabolism. Drugs Today, 42:39-64.

Fruchart JC, Staels B, Duriez P. 2001. The role of fibric acids in atherosclerosis. Curr Atheroscl Rep, 3:83-92.

Genest J, Frohlich J, Steiner G. 2004. Effect of fenofibrate-mediated increase in plasma homocysteine on the progression of coronary artery disease in type 2 diabetes mellitus. Am J Cardiol, 93:848-53.

Ginsberg HN, Bonds DE, Lovato LC, et al. 2007. Evolution of the lipid trial protocol of the Action to Control Cardiovascular Risk in Diabetes (ACCORD) trial. Am J Cardiol, 99(Suppl):56i-67i.

Gordon T, Castelli WP, Hjortland MC, et al. 1977. High density lipoprotein as a protective factor against coronary heart disease. The Framingham Study. Am J Med, 62:707-14.

Gordon DJ, Probstfield JL, Garrison RJ, et al. 1989. High-density lipoprotein cholesterol and cardiovascular disease. Four prospective American studies. Circulation, 79:8-15. 
Graham DJ, Staffa JA, Shatin D, et al. 2004. Incidence of hospitalized rhabdomyolysis in patients treated with lipid-lowering drugs. JAMA, 292:2585-90.

Grundy SM, Cleeman JI, Merz CNB, et al. 2004a. for the Coordinating Committee of the National Cholesterol Education Program. Implications or recent clinical trials for the National Cholesterol Education Program Adult Treatment Panel III guidelines. Circulation, 110:227-39.

Grundy SM, Hansen B, Smith SC Jr et al. 2004b. for the American Heart Association, the National Heart, Lung, and Blood Institute, and The American Diabetes Association. Clinical management of metabolic syndrome: report of the American Heart Association/National Heart, Lung, and Blood Institute/American Diabetes Association Conference on scientific issues related to management. Circulation, 109:551-6.

Grundy SM, Vega GL, Yuan Z, et al. 2005. Effectiveness and tolerability of simvastatin plus fenofibrate for combined hyperlipidemia (the SAFARI trial). Am J Cardiol, 95:462-8.

Guerin M, Bruckert E, Dolphin PJ, et al. 1996. Fenofibrate reduces plasma cholesteryl ester transfer from HDL to VLDL and normalizes the atherogenic dense LDL profile in combined hyperlipidemia. Arterioscler Thromb Vasc Biol, 16:763-72.

Gustavson LE, Schweitzer SM, Koehne-Voss S, et al. 2005. The effects of multiple doses of fenofibrate on the pharmacokinetics of pravastatin and its 3alpha-hydroxy isomeric metabolite. J Clin Pharmacol, 45:947-53.

Hottelart C, El Esper N, Rose F, et al. 2002. Fenofibrate increases creatinemia by increasing metabolic production of creatinine. Nephron, 92:536-41.

Jones PH, Davidson MH. 2005. Reporting rate of rhabdomyolysis with fenofibrate + statin versus gemfibrozil + any statin. Am J Cardiol, 95:120-2.

Kaneko T, Fuji S, Matsumoto A, et al. 2002. Induction of plasminogen activator inhibitor-1 in endothelial cells by basic fibroblast growth factor and its modification by fibric acid. Arterioscler Thromb Vasc Biol, 22:855-60.

Kastelein JJP, van der Steeg WA, Holme I, et al. 2008. Lipids, apolipoproteins, and their ratios in relation to cardiovascular events with statin treatment. Circulation, 117:3002-9.

Keating GM, Croom KF. 2007. Fenofibrate. A review of its use in primary dyslipidaemia, the metabolic syndrome and type 2 diabetes mellitus. Drugs, 67:121-53.

Keating GM, Ormrod D. 2002. Micronised fenofibrate. An updated review of its clinical efficacy in the management of dyslipidaemia. Drugs, 62:1909-44.

Keech T. 2006. American Heart Association Annual Scientific Sessions, Chicago, 2006.

Koh KK, Han SH, Quon MJ, et al. 2005. Beneficial effects of fenofibrate to improve endothelial dysfunction and raise adinopectin levels in patients with primary hypertriglyceridemia. Diabetes Care, 28:1419-24.

Liamis G, Bairaktari ET, Elisaf MS. 1999. Effect of fenofibrate on serum uric acid levels. Am J Kidney Dis, 34:594.

McBride PE. 2007. Triglycerides and risk for coronary heart disease. JAMA, 298:336-8.

McKenney JM, Farnier M, Lo KW, et al. 2006. Safety and efficacy of longterm co-administration of fenofibrate and ezetimibe in patients with mixed hyperlipidemia. J Am Coll Cardiol, 47:1584-7.

McKenney J, Jones M, Abby S. 2005. Safety and efficacy of colesevelam hydrochloride in combination with fenofibrate for the treatment of mixed hyperlipidemia. Curr Med Res Opin, 21:1403-12.

Maison P, Mennen L, Sapinho D, et al. 2002. A pharmacoepidemiological assessment of the effect of statins and fibrates on fibrinogen concentration. Atherosclerosis, 160:155-60.

Mardones P, Pilon A, Bouly M, et al. 2003. Fibrates down-regulate hepatic scavenger receptor class B type 1 protein expression in mice. J Biol Chem, 278:7884-90.
Martin PD, Dane AL, Shneck DW, et al. 2003. An open-label, randomized, three-way crossover trial of the effects of coadministration of rosuvastatin and fenofibrate on the pharmacokinetic properties of rosuvastatin and fenofibric acid in healthy male volunteers. Clin Ther, 25:459-71.

May HT, Anderson JL, Pearson RR, et al. 2008. Comparison of effects of simvastatin alone versus fenofibrate alone versus simvastatin plus fenofibrate on lipoprotein subparticle profiles in diabetic patients with mixed dyslipidemia (from the Diabetes and Combined Lipid Therapy Regimen study). Am J Cardiol, 101:486-9.

Mikael LG, Genest J Jr, Rozen R. 2006. Elevated homocysteine reduces apolipoprotein A-I expression in hyperhomocysteinemic mice and in males with coronary artery disease. Circ Res, 98:564-71.

Miller M, Cannon CP, Murphy SA, et al. 2008. for the PROVE IT-TIMI 22 Investigators. Impact of triglycerides levels beyond low-density lipoprotein cholesterol after acute coronary syndrome in the PROVE IT-TIMI 22 trial. J Am Coll Cardiol, 51:724-30.

Muhlestein JB, May HT, Jensen FR, et al. 2006. The reduction of inflammatory biomarkers by statin, fibrate, and combination therapy among diabetic patients with mixed dyslipidemia. The DIACOR (Diabetes and Combined Lipid Therapy Regimen) study. J Am Coll Cardiol, 48:396-401.

[NCEP] Executive summary of the third report of the National Cholesterol Education Program (NCEP) Expert Panel on Detection, Evaluation, and Treatment of High Blood Cholesterol in Adults (Adult Treatment Panel III). 2001. JAMA, 285:2486-97.

Nissen SE, Tuzcu EM, Schoenhagen P, et al. 2004. Effect of intensive compared with moderate lipid-lowering therapy on progression of coronary atherosclerosis. A randomized controlled trial. JAMA, 291:1071-80.

Nordestgaard BG, Benn M, Schnohr P, et al. 2007. Nonfasting triglycerides and risk of myocardial infarction, ischemic heart disease, and death in men and women. JAMA, 298:299-308.

Pan WJ, Gustavson LE, Achari R, et al. 2000. Lack of a clinically significant pharmacokinetic interaction between fenofibrate and pravastatin in healthy volunteers. J Clin Pharmacol, 40:316-23.

Paumelle R, Staels B. 2008. Cross-talk between statins and PPAR $\alpha$ in cardiovascular diseases: clinical evidence and basic mechanisms. Trends Cardiovasc Med, 18:73-8.

Playford DA, Watts GF, Best JD, et al. 2002. Effect of fenofibrate on brachial artery flow-mediated dilatation in type 2 diabetes mellitus. $\mathrm{Am} \mathrm{J}$ Cardiol, 90:1254-7.

Prueksaritanont T, Subramanian R, Fang X, et al. 2002b. Glucuronidation of statins in animals and humans: a novel mechanism of statin lactonization. Drug Metab Dispos, 30:505-12.

Prueksaritanont T, Tang C, Qiu Y, et al. 2002a. Effects of fibrates on metabolism of statins in human hepatocytes. Drug Metab Dispos, 30:1280-7.

Ridker PM, Rifai N, Cook NR, et al. 2005. Non-HDL-cholesterol, apolipoproteins A-I and B100, standard lipid measures, lipid ratios, and CRP as risk factors for cardiovascular disease in women. JAMA, 294:326-33.

Robins SJ, Collins D, Wittes JT, et al. 2001. for the VA-HIT Study Group. Relation of gemfibrozil treatment and lipid levels with major coronary events - VA-HIT: a randomized controlled trial. JAMA, 285:1585-91.

Rosenson RS, Kuskin AL, Wolff DA, et al. 2008. Fenofibrate reduces fasting and postprandial inflammatory responses among hypertriglyceridemia patients with the metabolic syndrome. Atherosclerosis, 198:381-8.

Rubins HB, Robins SJ, Collins D, et al. 2002. Diabetes, plasma insulin, and cardiovascular disease: subgroup analysis from the Department of Veterans Affairs high-density lipoprotein intervention trial (VA-HIT). Arch Intern Med, 162:2597-604.

Sarwar N, Danesh J, Eiriksdottir G, et al. 2007. Triglycerides and the risk of coronary heart disease: 10,158 incident cases among 262,525 participants in 29 Western prospective studies. Circulation, 115:450-8. 
Scott R, d'Emden M, Best J, et al. 2007. on behalf of the FIELD Investigators. Features of metabolic syndrome indentify individuals with type 2 diabetes mellitus at high risk for cardiovascular events and greater absolute benefits of fenofibrate. Circulation, 116(Suppl II):838.

Shek A, Ferrill MJ. 2001. Statin-fibrate combination therapy. Ann Pharmacother, 35:908-17.

Staels B, Dallongeville J, Auwerx J, et al. 1998. Mechanism of action of fibrates on lipid and lipoprotein metabolism. Circulation, 98:2088-93.

Stefanutti C, Bucci A, Di Giacomo S, et al. 2004. Efficacy, safety and tolerability of combined low-dose simvastatin-fenofibrate treatment in primary mixed hyperlipidaemia. Clin Drug Invest, 24:465-77.

Stone NJ, Bilek S, Rosenbaum S. 2005. Recent National Cholesterol Education Program Adult Treatment Panel III update: adjustments and options. Am J Cardiol, 96:53E-59E.

Syvanne M, Whittall RA, Turpeinen U, et al. 2004. Serum homocysteine concentrations, gemfibrozil treatment, and progression of coronary atherosclerosis. Atherosclerosis, 172:267-72.

Szapary PO, Rader DJ. 2004. The triglyceride-high density lipoprotein axis: an important target of therapy? Am Heart J, 148:211-21.

The ACCORD Study Group. 2007. Action to Control Cardiovascular Risk in Diabetes (ACCORD) trial: design and methods. Am J Cardiol, 99 (Suppl):21i-33i.

The FIELD study investigators. 2005. Effects of long-term fenofibrate therapy on cardiovascular events in 9795 people with type 2 diabetes mellitus (the FIELD Study):randomised controlled trial. Lancet, 366:1849-61.
The Task Force on Diabetes and Cardiovascular Diseases of the European Society of Cardiology (ESC) and the European Association for the Study of Diabetes (EASD). 2007. Guidelines on diabetes, pre-diabetes, and cardiovascular diseases: executive summary. Eur Heart J, 28:88-136.

Tribble DL, Farnier M, Macdonell G,et al. 2008. Effects of fenofibrate and ezetimibe, both as monotherapy and in coadministration, on cholesterol mass within lipoprotein subfractions and low-density lipoprotein peak particle size in patients with mixed hyperlipidemia. Metab Clin Experim, 57:796-801.

Tsimichodimos V, Bairaktari E, Elisaf M. 2002. Fibrate-induced increase in serum urea and creatinine levels. Nephrol Dial Transplant, 17:682.

Undas A, Brozek J, Szczeklik A. 2005. Homocysteine and thrombosis: from basic science to clinical evidence. Thromb Haemost, 94:907-15.

Vakkilainen J, Steiner G, Ansquer JC, et al. 2003. Relationships between low-density lipoprotein particle size, plasma lipoproteins, and progression of coronary artery disease: the Diabetes Atherosclerosis Intervention Study (DAIS). Circulation, 107:1733-7.

Vega GL, Ma PTS, Cater NB, et al. 2003. Effects of adding fenofibrate (200 mg/day) to simvastatin (10 mg/day) in patients with combined hyperlipidemia and metabolic syndrome. Am J Cardiol, 91:956-60.

Wierzbicki AS, Mikhailidis DP, Wray R, et al. 2003. Statin-fibrate combination therapy for hyperlipidaemia: a review. Curr Med Res Opin, 19:1-14.

World Health Statistics. 2008. www.who.int/whosis

Zambon A, Gervois P, Pauletto P, et al. 2006. Modulation of hepatic inflammatory risk markers of cardiovascular diseases by PPAR-alpha activators: clinical and experimental evidence. Arterioscler Thromb Vasc Biol, 26:977-86. 